\title{
Robust Nominal Model-Based Sliding Mode Robust Control for Vibration of Flexible Rectangular Plate
}

\author{
Jingyu Yang ${ }^{1}$, Guoping Chen ${ }^{2}$ \\ ${ }^{1}$ Department of Aeronautics and Astronautics, Shenyang Aerospace University, Shenyang 110136, China \\ ${ }^{2}$ The State Key Laboratory of Mechanics and Control for Mechanical Structures, Nanjing University of Aeronautics and Astronautics, \\ Nanjing 210016, China
}

\begin{abstract}
In this paper, we developed an approach for active vibration control of flexible rectangular plate structures using control theory. The flexible rectangular plate system is firstly modelled and simulated via a finite element method; and secondly, a new type of modelling method, and the state-space model are involved in the development of the equation of motion in state-space, which is efficiently used for the analysis of the system and design of control laws with a modern control framework. Then, the validity of the obtained new model is investigated by comparing the plate natural frequencies and forced vibration response analysis predicted by the finite element model with the calculated values obtained from new model. After validating the model, nominal model-based sliding mode robust MIMO controller is applied to the plate dynamics via the MATLAB/Simulink platform. The simulation results clearly demonstrate an effective vibration suppression capability that can be achieved using nominal model-based sliding mode robust MIMO controller.
\end{abstract}

Keywords: Flexible Rectangular Plate, Active Vibration Control, Modelling Method, Nominal Model-Based Sliding Mode Robust MIMO Controller

\section{Introduction}

Since the first earth orbiting satellite, Sputnik 1, was launched by the Soviet Union in 1957 , more and more lightweight materials and larger structures have been used in the field of aerospace, especially for antennas and solar panels in aircraft design. Many vibration problems have been caused due to the characteristics of lighter structures, including the following contents: low natural frequencies with high modal density, small material damping, geometric and material nonlinearities. Therefore, active vibration control method is the most effective for vibration control of flexible structures, and many scholars devoted much work to do research about active vibration control of flexible structures.

The AVC problem of flexible plate structures has attracted considerable attention during the last two decades. Many researchers proposed different control strategies for the purpose of AVC of flexible plate structures. $\mathrm{Hu}$ et al [1].applied LMI (Linear Matrix Inequality)-based robust control for AVC of a flexible plate structure. They used specific transformations of Lyapunov variable with appropriate linearizing transformations of the controller variables, which give rise to a tractable and practical LMI formulation of the vibration control problem. Based on LMI, a robust output feedback controller was designed to suppress the low-frequency vibrations caused by external disturbances. The simulation results showed that the proposed robust active control method is efficient for active vibration sup-pression. Other researches on the effectiveness of the robust robust control for AVC of the flexible structures have been addressed in [2]-[3].

Variable structure system (VSS) is based on the concept of an attractive manifold of the underlying state or error vector space on which the desired dynamic behavior is assured. These systems are a special class of nonlinear systems characterized by a discontinuous control action which changes structure upon reaching a sliding surface $s(s)=0$. A fundamental property of VSS is the sliding motion of the state on the attractive manifold. During this sliding motion the system has invariance

\footnotetext{
* Corresponding author e-mail: jingyu220@163.com
} 
properties, yielding motion which is independent of certain system parameter variations and disturbances. The concept of sliding mode control was first presented by K.D.Young [4] in 1978. Since then, many researchers employed the sliding mode control for different control problems such as robot motion control. However, a few papers can be found about the application of the sliding mode control for AVC of the flexible structures. Among developed control algorithms using the theory of VSS, several approaches have been considered. Some use the method of the hierarchy [4], others do use the Lyapunov stability method [5]-[6], and the linearizable method [7]-[8].

Based on the previously outlined literature, there is no published report in which the nominal model-based sliding mode robust MIMO control is used for the purpose of intelligent AVC of a flexible rectangular plate system. In this research, a nominal model-based sliding mode robust MIMO control strategy is applied to the problem of AVC of a rectangular flexible rectangular plate. First, the flexible rectangular plate system is modeled using the FEM method and new modelling method. Then, the validity of the obtained new model is investigated by comparing the plate natural frequencies, mode shape, static analysis and forced vibration response analysis predicted by the finite element model with the calculated values obtained from new model. After validating the model, nominal model-based sliding mode robust MIMO controller is applied to the plate dynamics via the MATLAB/Simulink platform. The algorithms were then coded in MATLAB to evaluate the performance of the control system. Disturbances were employed to excite the plate system at different excitation points and the controller ability to attenuate the vibration of observation point was investigated. The simulation results clearly demonstrate an effective vibration suppression capability that can be achieved using nominal model-based sliding mode robust MIMO controller.

\section{Modelling of Flexible Rectangular Plate System}

Cartesian coordinate system $(\mathrm{x}, \mathrm{y}, \mathrm{z})$ is introduced, consider a thin flexible rectangular plate of length a, along $\mathrm{x}$-axis, width, $\mathrm{b}$, along $\mathrm{y}$-axis and thickness, $\mathrm{h}$ along $\mathrm{z}$-axis. This condition is illustrated in Fig. 1. The quality and flexibility of plate structure is a continuous distribution, the system has an infinite number of degrees of freedom. To simplify the re-search and facilitate the calculation, construct spring-mass system and make the system discrete, the system is simplified as multi-freedom vibration system. After the process of discrete, the flexible rectangular plate is shown in Fig.2. $m_{j j}$ for $i \in\{1,2,3\}$ and $j \in\{1,2,3\}$ are masses; $k_{s t}$ for $s \in\{1,2,3,4,5\}$ and $t \in\{1,2,3\}$ are stiffness coefficients; $c_{r p}$ for $r \in\{1,2,3,4,5\}$ and $p \in\{1,2,3\}$ are

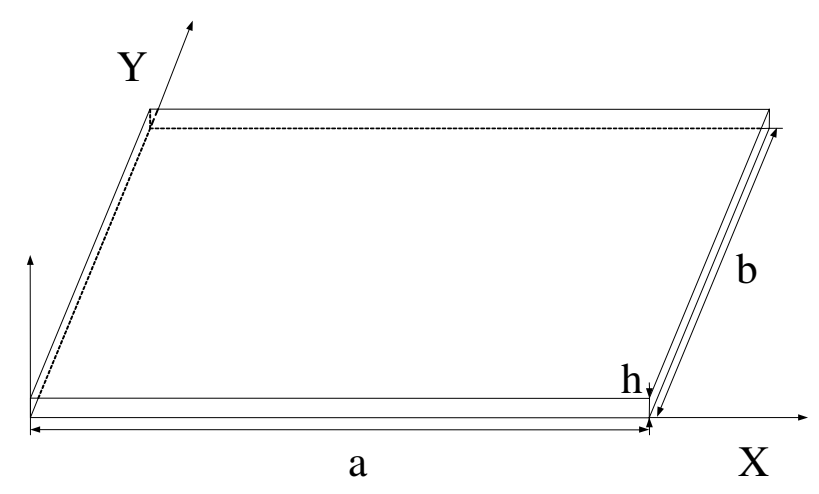

Fig. 1 A flexible rectangular plate

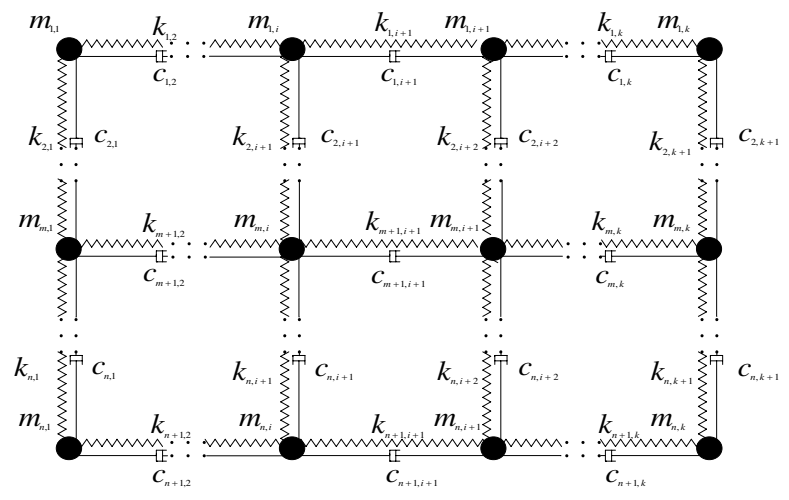

Fig. 2 A discrete flexible rectangular plate

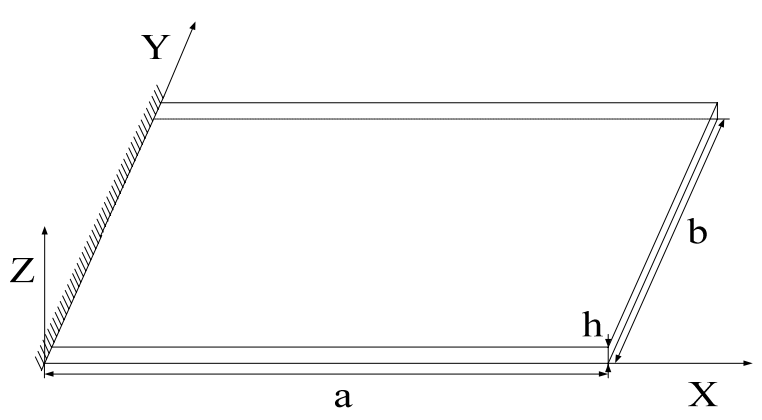

Fig. 3 A flexible cantilever plate

damping coefficients. $F_{11}$ is a concentrated force which is applied to $m_{j j}, \theta$ is generalized coordinate, $L$ is the length between the adjacent mass; $\nabla L$ is the variable value of $L$. 


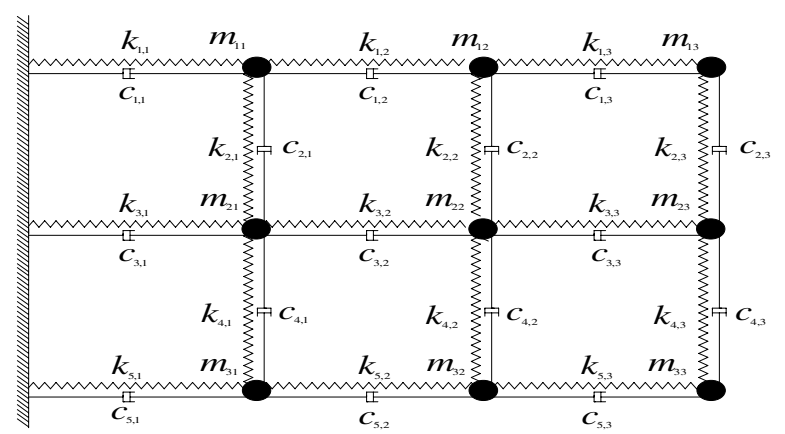

Fig. 4 A discrete flexible cantilever plate

$y$ is the elastic displacement of mass. This condition is illustrated in Fig.5.

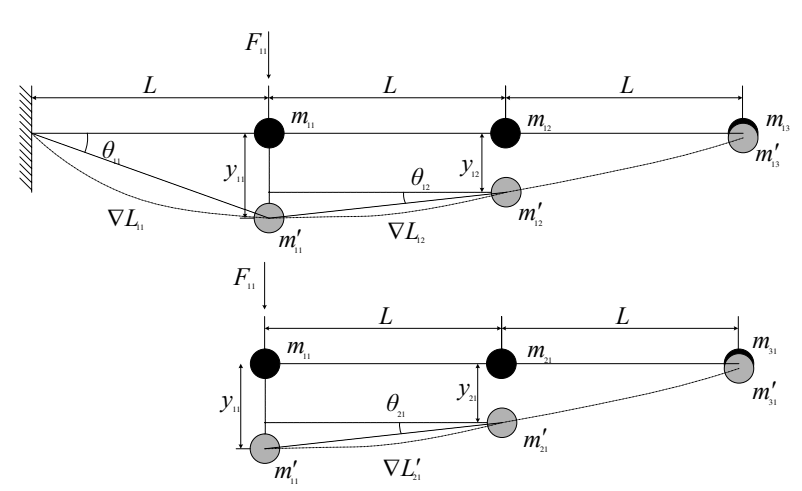

Fig. 5 Dynamic analysis of deformed plate

$$
\begin{gathered}
\sin \theta_{11}=\frac{y_{11}}{L+\Delta L_{11}}=\frac{\dot{y}_{11}}{\dot{L}+\Delta \dot{L}_{11}} \\
\sin \theta_{12}=\frac{y_{11}-y_{12}}{L+\Delta L_{12}}=\frac{\dot{y}_{11}-\dot{y}_{12}}{\dot{L}+\Delta \dot{L}_{12}} \\
\sin \theta_{21}=\frac{y_{11}-y_{21}}{L+\Delta L_{21}}=\frac{\dot{y}_{11}-\dot{y}_{21}}{\dot{L}+\Delta \dot{L}_{21}} \\
\Delta L_{11}<<L ; \Delta L_{12}<<L ; \Delta L_{21}<<L ; L=0 \\
\sin \theta_{11}=\frac{y_{11}}{L}=\frac{\dot{y}_{11}}{\Delta \dot{L}_{11}} ; \sin \theta_{12}=\frac{y_{11}-y_{12}}{L}=\frac{\dot{y}_{11}-\dot{y}_{12}}{\Delta \dot{L}_{12}}
\end{gathered}
$$

$$
\sin \theta_{21}=\frac{y_{11}-y_{21}}{L}=\frac{\dot{y}_{11}-\dot{y}_{21}}{\Delta \dot{L}_{21}}
$$

Letting

$$
\begin{gathered}
k_{11}^{\prime}=\left(k_{11}+k_{12}+k_{21}\right) / 3 ; \\
k_{12}^{\prime}=\left(k_{11}+k_{22}+k_{13}\right) / 3 ; k_{13}^{\prime}=\left(k_{12}+k_{23}\right) / 2 ; \\
k_{22}^{\prime}=\left(k_{21}+k_{12}+k_{23}+k_{32}\right) / 4 ; \\
k_{23}^{\prime}=\left(k_{22}+k_{13}+k_{33}\right) / 3 ; \quad k_{31}^{\prime}=\left(k_{31}+k_{21}+k_{32}\right) / 3 ; \\
k_{32}^{\prime}=\left(k_{31}+k_{22}+k_{33}\right) / 3 ; \quad k_{33}^{\prime}=\left(k_{32}+k_{23}\right) / 2
\end{gathered}
$$

Supposed:

$$
\begin{gathered}
k_{11}^{\prime} \cdot \frac{\nabla L_{11}}{L} \approx k_{11}^{\prime} \cdot \frac{\nabla L_{12}}{L} \approx k_{11}^{\prime} \cdot \frac{\nabla L_{21}^{\prime}}{L} \approx k_{11}^{\prime} \cdot \frac{\nabla L_{1}}{L} ; \\
k_{12}^{\prime} \cdot \frac{\nabla L_{11}}{L} \approx k_{12}^{\prime} \cdot \frac{\nabla L_{22}}{L} \approx k_{12}^{\prime} \cdot \frac{\nabla L_{13}^{\prime}}{L} \approx k_{12}^{\prime} \cdot \frac{\nabla L_{2}}{L} ; \\
k_{13}^{\prime} \cdot \frac{\nabla L_{12}}{L} \approx k_{13}^{\prime} \cdot \frac{\nabla L_{23}}{L} \approx k_{13}^{\prime} \cdot \frac{\nabla L_{3}}{L} ; \\
k_{22}^{\prime} \cdot \frac{\nabla L_{21}}{L} \approx k_{21}^{\prime} \cdot \frac{\nabla L_{22}}{L} \approx k_{21}^{\prime} \cdot \frac{\nabla L_{11}^{\prime}}{L} \approx k_{21}^{\prime} \cdot \frac{\nabla L_{31}^{\prime}}{L} \approx k_{21}^{\prime} \cdot \frac{\nabla L_{4}}{L} ; \\
k_{23}^{\prime} \cdot \frac{\nabla L_{12}}{L} \approx k_{22}^{\prime} \cdot \frac{\nabla L_{23}^{\prime}}{L} \approx k_{22}^{\prime} \cdot \frac{\nabla L_{32}^{\prime}}{L} \approx k_{22}^{\prime} \cdot \frac{\nabla L_{5}}{L} ; \\
k_{31}^{\prime} \cdot \frac{\nabla L_{31}}{L} \approx \frac{\nabla L_{13}}{L} \approx k_{31}^{\prime} \cdot \frac{\nabla L_{21}}{L} \approx \frac{\nabla L_{33}^{\prime}}{L} \approx k_{23}^{\prime} \cdot \frac{\nabla L_{6}}{L} ; \\
k_{32}^{\prime} \cdot \frac{\nabla L_{31}}{L} \approx k_{32}^{\prime} \cdot \frac{\nabla L_{22}^{\prime}}{L} \approx k_{32}^{\prime} \cdot \frac{\nabla L_{33}^{\prime}}{L} \approx k_{32}^{\prime} \cdot \frac{\nabla L_{8}}{L} ; \\
L
\end{gathered}
$$

Letting

$$
k_{11}^{\prime} \cdot \frac{\nabla L_{1}}{L} \approx K_{11} ; k_{12}^{\prime} \cdot \frac{\nabla L_{2}}{L} \approx K_{12} ; k_{21}^{\prime} \cdot \frac{\nabla L_{4}}{L} \approx K_{21} ;
$$$$
k_{22}^{\prime} \cdot \frac{\nabla L_{5}}{L} \approx K_{22} ; \quad k_{23}^{\prime} \cdot \frac{\nabla L_{6}}{L} \approx K_{23} ; \quad k_{31}^{\prime} \cdot \frac{\nabla L_{7}}{L} \approx K_{31} ;
$$$$
k_{32}^{\prime} \cdot \frac{\nabla L_{8}}{L} \approx K_{32} ; k_{33}^{\prime} \cdot \frac{\nabla L_{9}}{L} \approx K_{33} ; c_{11}^{\prime}=C_{11} ;
$$ 


$$
\begin{gathered}
c_{13}^{\prime}=C_{13} ; c_{22}^{\prime}=C_{22} ; c_{23}^{\prime}=C_{23} ; c_{31}^{\prime}=C_{31} ; \\
c_{32}^{\prime}=C_{32} ; c_{33}^{\prime}=C_{33}
\end{gathered}
$$

We now apply Newton's second law of motion to the mass $m_{j j}=m$ for $i \in\{1,2,3, \ldots, 7\}$ and $j \in\{1,2,3, \ldots, 7\}$

$$
M_{11} \ddot{y}_{11}+C_{11} \cdot\left[3 \dot{y}_{11}-\dot{y}_{12}-\dot{y}_{21}\right]+K_{11}\left[3 y_{11}-y_{12}-y_{21}\right]=F_{11}
$$

$$
M_{12} \ddot{y}_{12}+C_{12} \cdot\left[3 \dot{y}_{11}-\dot{y}_{12}-\dot{y}_{21}\right]+K_{12}\left[3 y_{12}-y_{13}-y_{22}-y_{11}\right]=F_{12}
$$

$$
M_{13} \ddot{y}_{13}+C_{13} \cdot\left[3 \dot{y}_{11}-\dot{y}_{12}-\dot{y}_{21}\right]+K_{13}\left[2 y_{13}-y_{12}-y_{23}\right]=F_{13}
$$

$$
M_{21} \ddot{y}_{21}+C_{21} \cdot\left[3 \dot{y}_{11}-\dot{y}_{12}-\dot{y}_{21}\right]+K_{21}\left[4 y_{21}-y_{11}-y_{31}-y_{22}\right]=F_{21}
$$

$$
M_{22} \ddot{y}_{22}+C_{22} \cdot\left[3 \dot{y}_{11}-\dot{y}_{12}-\dot{y}_{21}\right]+K_{22}\left[4 y_{22}-y_{21}-y_{12}-y_{32}\right]=F_{22}
$$

$$
M_{23} \ddot{y}_{23}+C_{23} \cdot\left[3 \dot{y}_{11}-\dot{y}_{12}-\dot{y}_{21}\right]+K_{23}\left[3 y_{23}-y_{13}-y_{33}-y_{22}\right]=F_{23}
$$

$$
M_{31} \ddot{y}_{31}+C_{31} \cdot\left[3 \dot{y}_{11}-\dot{y}_{12}-\dot{y}_{21}\right]+K_{31}\left[3 y_{31}-y_{32}-y_{21}\right]=F_{31}
$$

$$
M_{32} \ddot{y}_{32}+C_{32} \cdot\left[3 \dot{y}_{11}-\dot{y}_{12}-\dot{y}_{21}\right]+K_{32}\left[3 y_{32}-y_{33}-y_{22}-y_{31}\right]=F_{32}
$$

$$
M_{33} \ddot{y}_{33}+C_{33} \cdot\left[3 \dot{y}_{11}-\dot{y}_{12}-\dot{y}_{21}\right]+K_{33}\left[2 y_{33}-y_{32}-y_{23}\right]=F_{33}
$$

As a convention, we denote a dot as a first derivative with respect to time(i.e., $\dot{y}=d x / d t$ ), and a double dot as a second derivative with respect to time (i.e., $\ddot{y}=d^{2} x / d t^{2}$ ). Let $n_{d}$ be a number of degrees of freedom of the system (linearly independent coordinates describing the finite-dimensional structure), let $r$ be a number of outputs, and let $s$ be a number of inputs. A flexible structure in nodal coordinates is represented by the following second-order matrix differential equation:

$$
[M][\ddot{Y}]+[P][\dot{Y}]+[K][Y]=[L][F]
$$

In this equation $X$ is the $n_{d} \times 1$ nodal displacement vector; $\dot{y}$ is the $n_{d} \times 1$ nodal velocity vector; $\ddot{y}$ is the $n_{d} \times 1$ nodal acceleration vector; $F$ is the $s \times 1$ input vector; $[M]$ is the mass matrix, $n_{d} \times n_{d} ;[P]$ is the damping matrix, $n_{d} \times n_{d} ;[K]$ is the stiffness matrix, $n_{d} \times n_{d} ;[L]$ is input matrix, $n_{d} \times s$;. The mass matrix is positive definite (all its eigenvalues are positive), and the stiffness and damping matrices are positive semi-definite (all their eigenvalues are nonnegative).

\section{Modelling of Flexible Cantilever Plate}

The idea of the paralleled semi-extension rule based algorithm is as follows. Firstly, the algorithm decomposes the maximum terms space of the clause set into several partial maximum terms spaces, which convert the SAT problem of the clause set into the SAT problem of the partial maximum terms spaces. If there is a certain partial maximum terms space that is satisfiable, then the clause set is satisfiable. If all the partial maximum terms spaces are unsatisfiable, then the clause set is unsatisfiable. In other words, the clause set is satisfiable. In the following, the concept of the partial maximum terms space will be given. Finite element analysis for $10 \mathrm{~m} \times 10 \mathrm{mplate}, \rho=7800 \mathrm{~kg} / \mathrm{m} 3$. Thickness is $0.001 \mathrm{~m}$. This condition is illustrated in Fig. 6. Considering

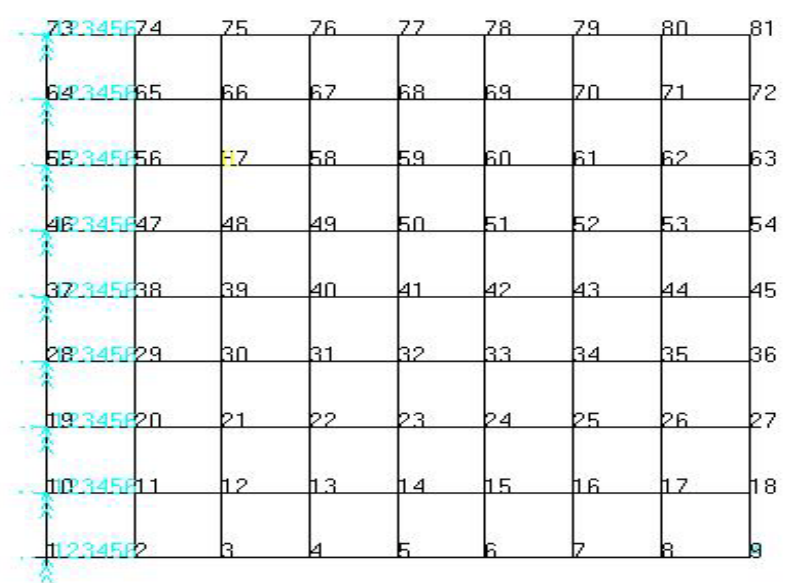

Fig. 6 Finite element model

flowchart of modelling method (Fig.7.), we have $[M],[P]$, $[K],[L]$

\section{Validity of the New Model}

A. Natural frequency comparison analysis The results of natural frequency contrastive analysis are shown in Table.1.

Table.1. Contrastive Analysis Result

\begin{tabular}{cccccc}
\hline Natural Frequence & 1 & 2 & 3 & 4 & 5 \\
FEA Reslut & 0.17 & 0.41 & 1.06 & 1.35 & 1.54 \\
\hline New Model & 0.17 & 0.41 & 1.06 & 1.35 & 1.54 \\
Absolute Error & 0.06 & 0.03 & 0.06 & 0.06 & 0.43 \\
Natural Frequence & 6 & 7 & 8 & 9 & \\
FEA Reslut & 2.71 & 3.19 & 3.32 & 3.70 & \\
New Model & 2.82 & 2.94 & 3.29 & 3.61 & \\
Absolute Error & 0.11 & 0.11 & 0.03 & 0.09 & \\
\hline
\end{tabular}




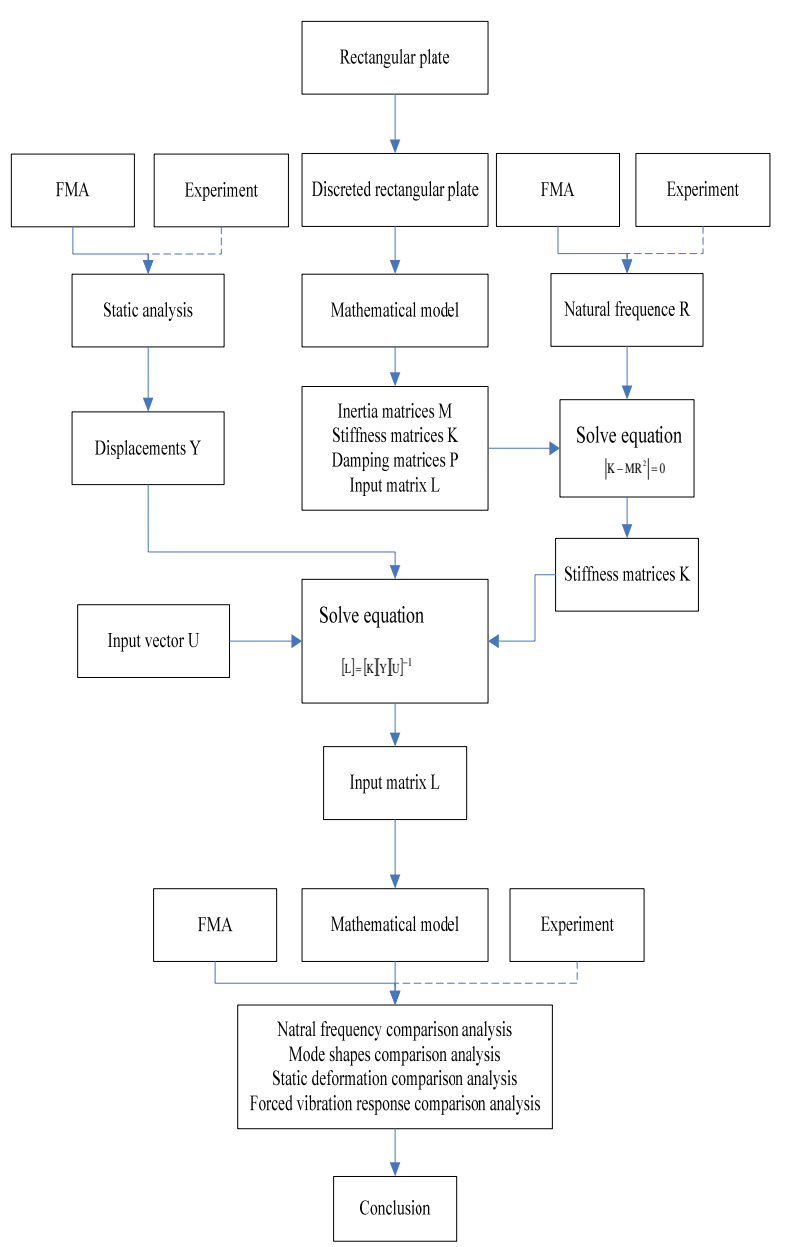

Fig. 7 Modelling method

B. Forced vibration response analysis When system is excited by a harmonic force, the vibration response of 39th node is shown by Fig. 8 .

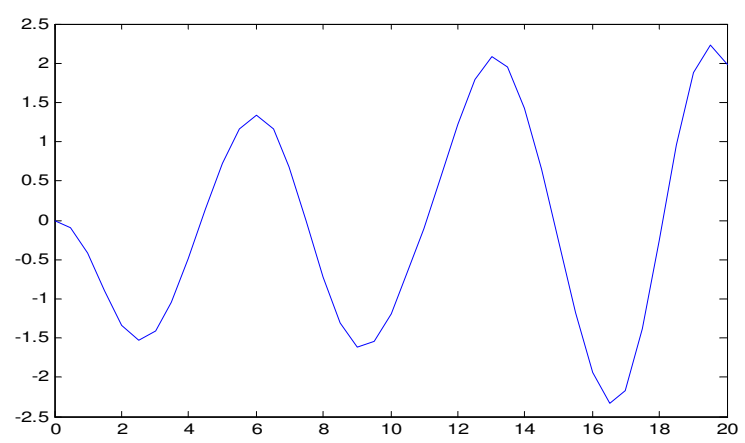

(a)

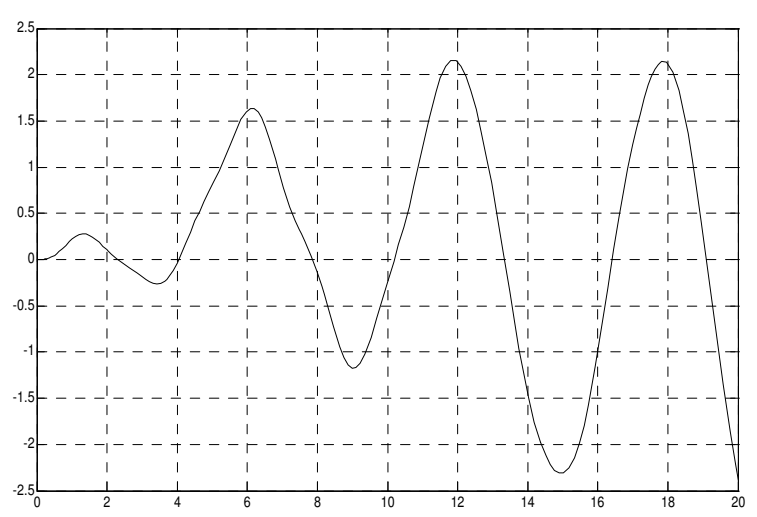

(b)

Fig. 8 Vibration response of flexible rectangular plate system (Acting point of force is 39 node) (a) Result of FEM model (b) Result of New model

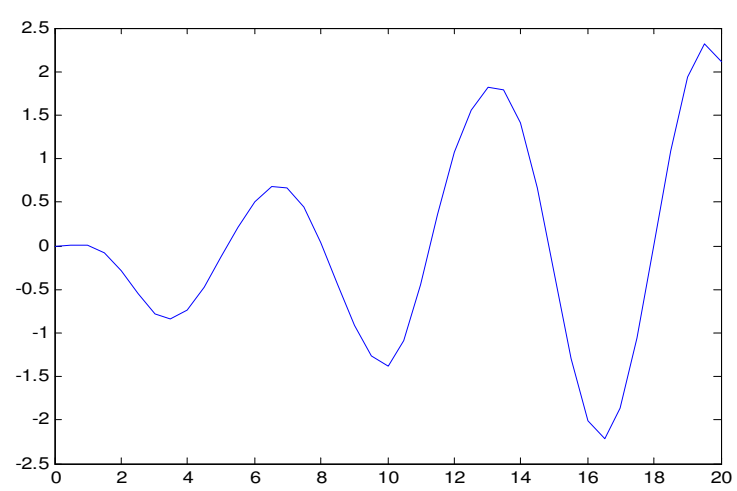

(a)

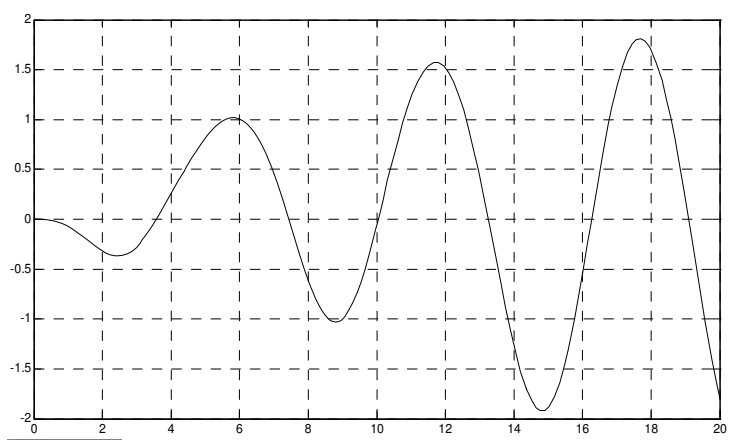

(b) 
Fig. 9 Vibration response of flexible rectangular plate system (Acting point of force is 57 node) (a) Result of FEM model (b) Result of New model

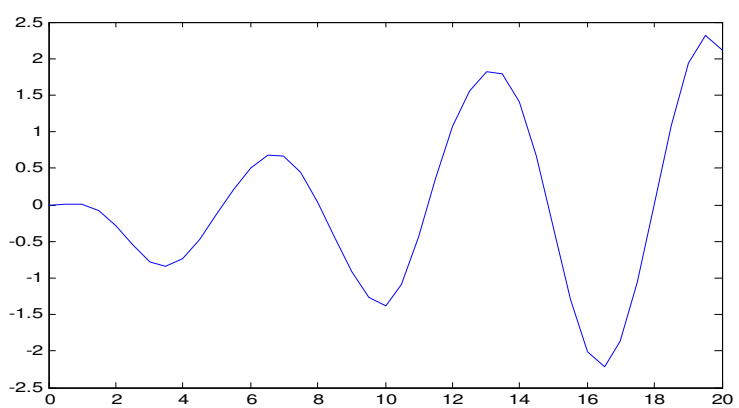

(a)

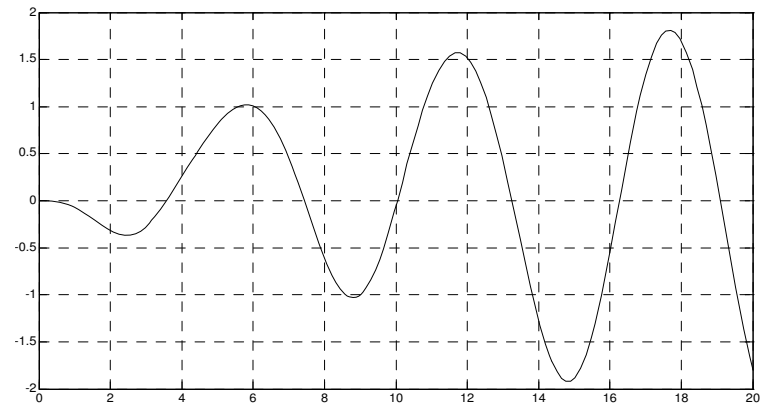

(b)

Fig. 10 Vibration response of flexible rectangular plate system (Acting point of force is 21 node) (a) Result of FEM model (b) Result of New model

\section{Nominal Model-Based Sliding Mode Robust MIMO Controller Design}

$$
M(q) \ddot{q}+C(q, \dot{q}) \dot{q}+K(q)=\tau+w
$$

where $q=\left[q_{1}, \ldots q_{n}\right]^{T}$ is an $n \times 1$ vector of node position, $\dot{q}=\left[\dot{q}_{1}, \ldots \dot{q}_{n}\right]^{T}$ is an $n \times 1$ vector of node velocity, $\ddot{q}=\left[\ddot{q}_{1}, \ldots \ddot{q}_{n}\right]^{T}$ is an $n \times 1$ vector of node acceleration, $\tau$ is an $n \times 1$ vector of the control input, $[M(q)]$ is an $n \times n$ inertial matrix, $[P(q, \dot{q})]$ is an $n \times n$ damping matrix and
$[K(q)]$ is an $n \times n$ stiffness matrix. The inertial matrix is symmetric and positive de-finite. It is also bounded as a function of $q: \mu_{1} I \leq \mathrm{M}(\mathrm{q}) \leq \mu_{2} I, m_{1} \leq \mathrm{M}(\mathrm{q}) \leq m_{2}$. The stiffness matrix $K(q)$ is bounded as a function of $q: K(q) \leq g_{h}$, where $g_{h}$ is a function of $q$. For simplification, $M(q), P(q, \dot{q})$ and $K(q)$ are written as $M, P$ and $K$,respectively. It is noticed that $M, P$ and $K$ are only partly known and therefore exists uncertainly in the system model. $\omega$ disturbance vector. The control objective is to drive the node position $q$ to the desired position $q_{d}$. Define the tracking error

$$
e=q-q_{d}
$$

Define the sliding surface

$$
S=\dot{e}+C e, \quad C=\operatorname{diag}\left(c_{1}, \ldots, c_{n}\right), \quad c_{i}>0
$$

To prove the stability of the system, choose the Lyapunov function candidate to be

$$
V=\frac{1}{2} S^{T} M S
$$

Consider the derivative of $V$

$$
\begin{aligned}
\dot{V} & =\frac{1}{2} S^{T} \dot{M} S+S^{T} M \dot{S}=S^{T} M(\ddot{e}+C \dot{e}) \\
& =S^{T}\left[M\left(\ddot{q}_{d}-\ddot{q}\right)+M C \dot{e}\right] \\
& =S^{T}\left[M \ddot{q}_{d}+C \dot{q}+K(q)-w-T+M C \dot{e}\right] \\
& =S^{T}\left[M\left(\ddot{q}_{d}+C \dot{e}\right)+C \dot{q}+K(q)-w-T\right]
\end{aligned}
$$

Choose the control input $T$ :

$$
T=M_{0}\left(\ddot{q}_{d}+C \dot{e}\right)+C_{0} \dot{q}+K_{0}(q)-w_{0}+T \operatorname{sgn}(S)
$$

where, $C_{0}, K_{0}$ and $\omega_{0}$ are the estimations of $M, P$ and $K$ and $\omega$ respectively, $\Delta M=M-M_{0}, \Delta B=B-B_{0}, \Delta K=K-K_{0}, \Delta \omega=\omega$ $\omega_{0}$, In this case, $\dot{V}(21)$ can be rewritten as

$$
\dot{V}=S^{T}\left[\Delta M\left(\ddot{q}_{d}+C \dot{e}\right)+\Delta C \dot{q}+\Delta K(q)-\Delta w\right]-\Gamma|S|
$$

where $\Gamma=\operatorname{diag}\left(\gamma_{1}, \gamma_{2}, \ldots \gamma_{n}\right),\left(\gamma_{i}>0\right)$. Let

$$
\gamma_{i}>|\Delta M|_{\max }\left|\dot{q}_{d}+C e\right|+|\Delta C|_{\max } \dot{q}+|\Delta w|_{\max }+|\Delta K|
$$

This yields

$$
\dot{V} \leq 0
$$

\section{Simulation Example}

Considering $[M],[P],[K],[L]$ and letting $M(q)=[M]$, $C(q, \dot{q})=[P], K(q)=[K][Y], \tau=[L][F]$ 


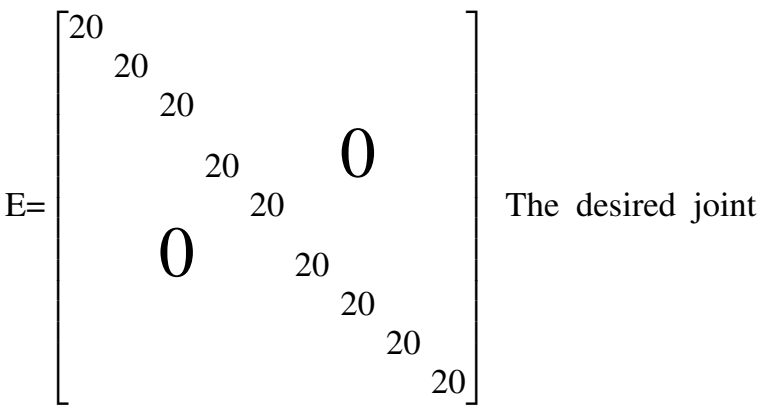

trajectory is given by $\mathrm{q}_{d}=\sin (t) ; q 2_{d}=\sin (t) ; q 3_{d}=$ $\sin (t) ; q 4_{d}=\sin (t) ; q 5_{d}=\sin (t) ; q 6_{d}=\sin (t) ; q 7_{d}=$ $\sin (t) ; q 8_{d}=\sin (t) ; q 9_{d}=\sin (t)$;

The displacements and velocities are chosen as

$x=\left[y_{1} ; \dot{y}_{1} ; y_{2} ; \dot{y}_{2} ; y_{3} ; \dot{y}_{3} ; y_{4} ; \dot{y}_{4} ; y_{5} ; \dot{y}_{5} ; y_{6} ; \dot{y}_{6} ; y_{7} ; \dot{y}_{7} ; y_{8} ; \dot{y}_{8} ; y_{9} ; \dot{y}_{9}\right]$

The initial displacements and velocities are chosen as $x_{0}$

$0.001[0.6 ; 0.3 ; 0.5 ; 0.5 ; 0.6 ; 0.3 ; 0.5 ; 0.5 ; 0.6 ; 0.3 ; 0.5 ; 0.5$; $0.6 ; 0.3 ; 0.5 ; 0.5 ; 0.5 ; 0.5]$

Using control laws (22), Fig.11 shows the simulation model. Fig.12 shows position tracking of 57 node , 59 node, 61 node, 39 node, 41 node, 43 node, 21 node, 23 node, 25 node respectively. Fig.13 shows control law output results.

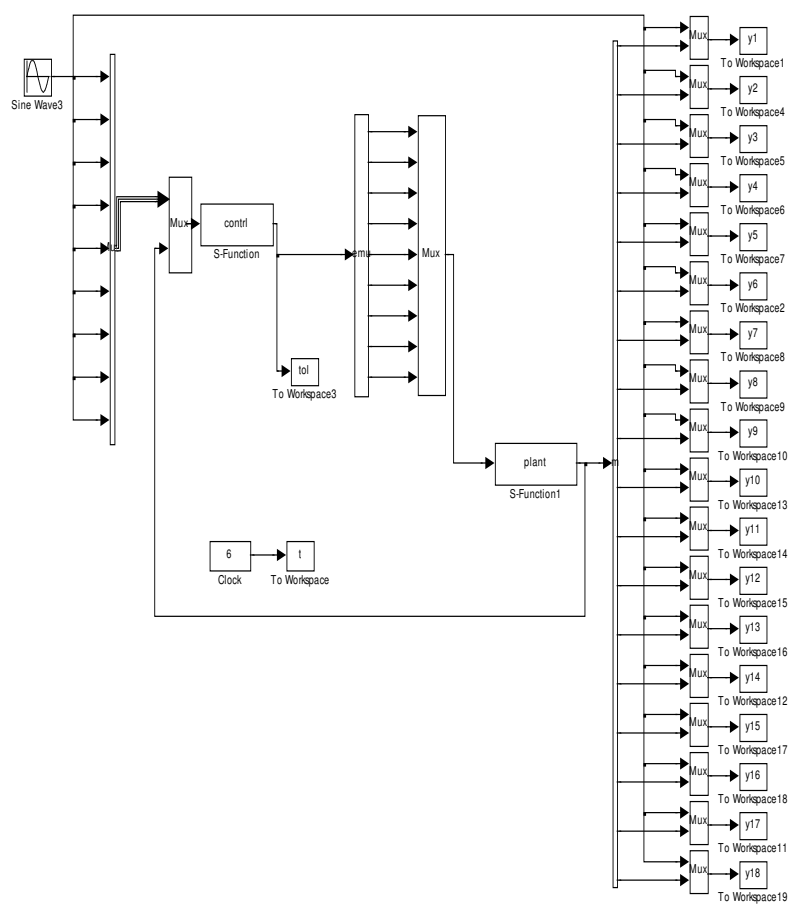

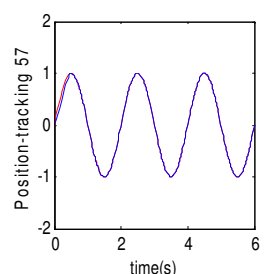
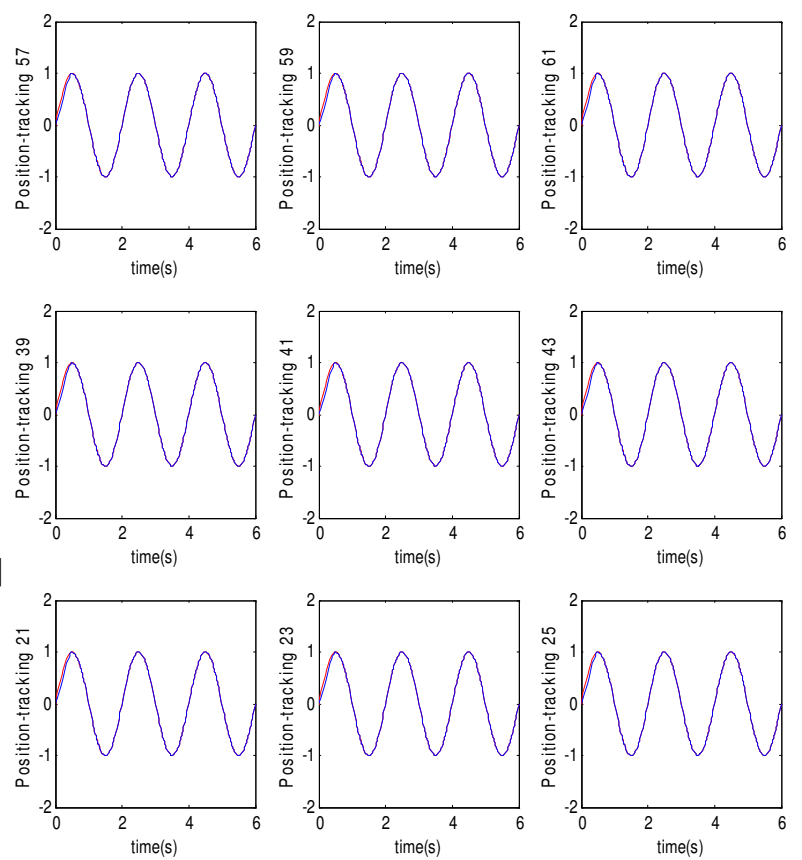

Fig. 12 Position tracking of 57 node, 59 node, 61 node, 39 node, 41 node, 43 node, 21 node, 23 node, 25 node respectively.
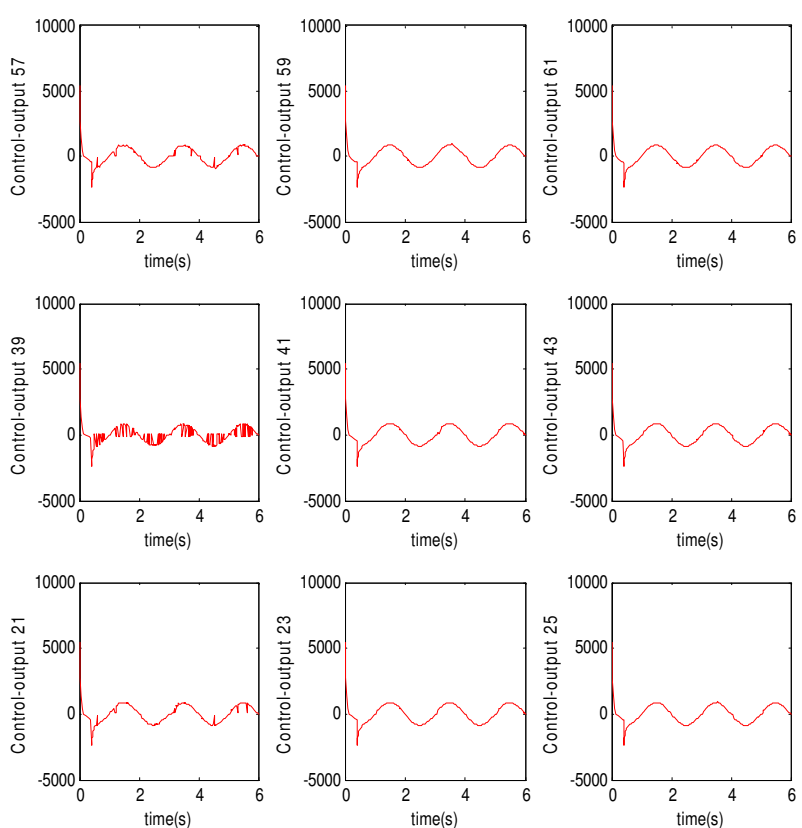

Fig. 13 Control law output results

Fig. 11 Simulation model 


\section{Perspective}

Nominal model-based sliding mode robust MIMO control strategy for the active vibration control of a flexible rectangular plate structure was developed. It was shown that the new modelling method was a kind of development with respect to the plant modelling theory of current control theory. It provides theoretical basis for low order controller design of high order plant with unknown parameters, adaptive controller design and intelligent controller design. It also brings about great convenience for engineering design. The first nine natural frequencies, forced vibration response analysis of the flexible rectangular plate structure considered in this study were pre-dicted accurately and compared by the FEM method and new modelling method and thus, the validity of the proposed new model was confirmed. A nominal model-based sliding mode robust MIMO controller was then employed to attenuate the unwanted vibration of a rectangular flexible plate system simulated using the MATLAB/Simulink platform. The simulation results demonstrated the effectiveness of the proposed control technique. Future works will be directed towards the development of an experimental rig to validate the theoretical results obtained in the study.

\section{Acknowledgement}

The work was supported by the Funding for Outstanding Doctoral Dissertation in NUAA (BCXJ10-01), Funding of Jiangsu Innovation Program for Graduate Education (CX10B $\left.{ }_{0} 89 Z\right)$, NUAAResearchF unding(No.NS20100060).

\section{References}

[1] . Hu, G. Ma, C. Li, Active vibration control of a ?exible plate structure using LMI-based output feedback control law, in: Proceedings of the 5th World Congress on Intelligent Control and Automation, China, 2004, pp. 738-742.

[2] .N. Kar, T. Miyakura, K. Seto, Bending and torsional vibration control of a ?exible plate structure using based robust control law, IEEE Trans.Control Syst. Technol. 8 (3) (2000) 545-553.

[3] . Xianmin, Sh. Changjian, A.G. Erdman, Active vibra-tion controller design and comparison study of flexible linkage mechanism systems, J. Mech.Mach. Theory 37 (2002) 985997.

[4] . Harashima, H. Hashimoto, and K. Maruyama, "Prac-tical robust control of robot arm using variable structure system," in Proc. Int. Conf. Robotics Automat.,1986, pp. 532-539.

[5] .Bailey and A. Arapostathis, "Simple sliding mode control scheme applied to robot manipulator," Int. J. Contr., vol. 45, pp. 1197-1209, 1987.

[6] . W. Wijesoma and R. J. Richards, "Robust trajectory following of robot using computed torque structurewith VSS,” Int. J. Contr., vol. 52 ,no.4, 935-962.
[7] .C. Fu and T.L.Liao, "Globally stable robust tracking of nonlinear system using variable structure control and with application to a robotic manipulator," IEEE Trans. Automat.Contr., vol.35, pp. 1345-1350, 1990.

[8].L. Pei and Q. J. Zhou, "Variable structure control of linearizable systems with applications to robot manipula-tor," in Proc. IEEE Int.Conf. Robotics Automat., 1991. 101-109, 1978.52, pp. 935-962,1991.

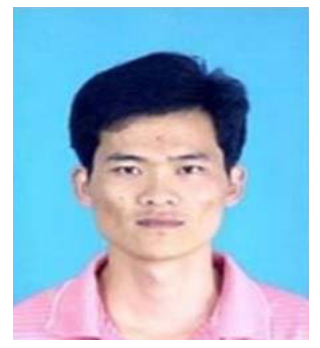

Jingyu Yang received the B.S. degree in Mechanical Engineering from the University of Science and Technology Liaoning, the M.S. degree in Mechanical Engineering from Tianjin University of Technology and Education, and the Ph.D. degree in Engineering Mechanics from Nanjing University of Aeronautics and Astronautics. Since August 2009, he has been with the College of Aerospace, Nanjing University of Aeronautics and Astronautics, where he became a Ph.D candidate April 2009. His research interests on dynamics of optimization, simulation and intelligent control. Corresponding author of this paper. E-mail: jingyu220@163.com.

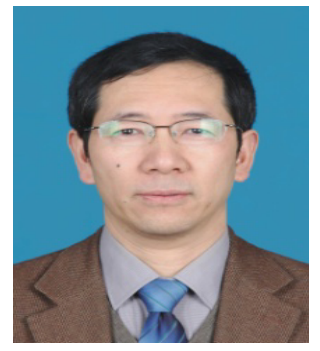

Guoping Chen $\mathrm{He}$ received the B.S. degree from the Department of Mechanics, Zhejiang University, in 1982, and the M.S. degree from the Department of Mechanics, Zhejiang University, in 1984, and the Ph.D. degree from the Department of Mechanics, Zhejiang University, in 1988. From August 1996 to August 1998, he was a visiting scholar in British Columbia University. From October 27,2011, he has served as vice chairman and secretary general of the seventh council of the china institute of vibration engineering. 